\title{
The biological basis and function of GNAS mutation in pseudomyxoma peritonei: a review
}

\author{
Yu-Lin $\operatorname{Lin}^{1} \cdot \operatorname{Ru} M a^{1} \cdot \operatorname{Yan} \mathrm{Li}^{1}$ (1)
}

Received: 9 June 2020 / Accepted: 10 July 2020 / Published online: 22 July 2020

(c) The Author(s) 2020

\begin{abstract}
Purpose Pseudomyxoma peritonei (PMP) is a rare clinical malignancy syndrome characterized by the uncontrollable accumulation of copious mucinous ascites in the peritoneal cavity, resulting in "jelly belly". The mechanism of tumor progression and mucin hypersecretion remains largely unknown, but GNAS mutation is a promising contributor. This review is to systemically summarize the biological background and variant features of GNAS, as well as the impacts of GNAS mutations on mucin expression, tumor cell proliferation, clinical-pathological characteristics, and prognosis of PMP.

Methods NCBI PubMed database (in English) and WAN FANG DATA (in Chinese) were used for literature search. And NCBI Gene and Protein databases, Ensembl Genome Browser, COSMIC, UniProt, and RCSB PDB database were used for gene and protein review.

Results GNAS encodes guanine nucleotide-binding protein $\alpha$ subunit (Gs $\alpha$ ). The mutation sites of GNAS mutation in PMP are relatively stable, usually at Chr20: 57,484,420 (base pair: C-G) and Chr20: 57,484,421 (base pair: G-C). Typical GNAS mutation results in the reduction of GTP enzyme activity in Gs $\alpha$, causing failure to hydrolyze GTP and release phosphoric acid, and eventually the continuous binding of GTP to Gs $\alpha$. The activated Gs $\alpha$ could thus continuously promote mucin secretion through stimulating the cAMP-PKA signaling pathway, which is a possible mechanism leading to elevated mucin secretion in PMP.

Conclusion GNAS mutation is one of the most important molecular biological features in PMP, with major functions to promote mucin hypersecretion.
\end{abstract}

Keywords Pseudomyxoma peritonei $\cdot G N A S \cdot$ Gene mutation $\cdot$ Signaling pathway $\cdot$ Mucin

\section{Introduction}

Pseudomyxoma peritonei (PMP) is a rare clinical malignancy syndrome usually caused by the perforation of appendiceal mucinous tumor and the "redistribution phenomenon" of mucus and tumor cells, with an incidence of $1-2 /$ million (Mittal et al. 2017; Smeenk et al. 2008). PMP is characterized by a large volume of mucinous ascites, multiple peritoneal implantations, omental cake, and ovarian involvement in women macroscopically, and abundant mucus pools microscopically. The chronic and uncontrollable mucus

Yan $\mathrm{Li}$

liyansd2@163.com

1 Department of Peritoneal Cancer Surgery and Pathology, Beijing Shijitan Hospital, Capital Medical University, No. 10 Tieyi Road, Yangfangdian Street, Haidian District, Beijing 100038, China accumulation is one of the major clinical features of PMP (O'Connell et al. 2002a, b), which gradually leads to intraperitoneal organ adhesion, bowel obstruction, malnutrition, and eventually cachexia and death. Aggressive cytoreductive surgery (CRS) combined with hyperthermic intraperitoneal chemotherapy (HIPEC) could bring significant survival benefit to PMP (Chua et al. 2012; Li et al. 2018), and has been recommended by Peritoneal Surface Oncology Group International (PSOGI) as the standard treatment of PMP ( $\mathrm{Li}$ et al. 2014, 2019).

Although treated with CRS plus HIPEC, patients frequently suffered from relapse, presenting aggravated "jelly belly". One of the difficulties in studying PMP is the scarcity of knowledge in the fundamental molecular mechanisms underlying mucus hypersecretion. It has been reported that Kirsten rat sarcoma viral oncogene homolog $(K R A S)$ and guanine nucleotide-binding protein alpha subunit (GNAS) are two of the most frequently detected variants in PMP, and 
GNAS mutation plays an important role in the regulation of mucin expression (Bradbury 2000; Jarry et al. 1994; Nishikawa et al. 2013). To have a better insight into the role of GNAS gene in PMP, we systemically reviewed the biological background of GNAS, current studies concerning the variant feature of GNAS, the impacts of GNAS mutations on mucin expression, tumor cell proliferation, and clinical-pathological characteristics and prognosis.

\section{The biological background of GNAS gene}

\section{Basic structure and function}

The GNAS gene is located at chromosome 20q13.32 (chromosome 20: 57,414,773-57,486,247), which also names GNAS complex locus (Fig. 1a), consisting of 13 exons and 12 introns. GNAS is responsible for the encoding of stimulatory guanine nucleotide-binding protein (G protein) $\alpha$ subunit (Gs $\alpha$ ), which transduces signals from $G$ protein-coupled receptors (GPCR) to adenyl cyclase (AC), and finally regulates the expression of cyclic adenosine monophosphate (cAMP).

\section{DNA transcription and translation}

The promoter region of Gs $\alpha$ is located at the $\mathrm{CpG}$ island upstream of exon 1, which is usually unmethylated in alleles of both parental origins (Bird 1986; Gardiner-Garden and Frommer 1987). It was reported by Mantovani et al. (2002) and Germain-Lee et al. (2005) that Gs $\alpha$ imprinted with tissue-specific pattern in kidney cortex, thyroid gland, pituitary gland, and ovary, which is mainly maternally expressed. There are four kinds of alternative promoter regions upstream of Gs $\alpha$ exon 1 (Weinstein et al. 2001): (1) promoter 1 , about $49 \mathrm{~kb}$ upstream of Gs $\alpha$ exon 1 , encodes neuroendocrine secretory protein 55 (NESP55). The coding sequence is within the upstream of Gs $\alpha$ exon 1, leaving exon 2-13 untranslated region; (2) promoter 2, about 2-3 kb upstream of XL exon, initiates NESP55 exon transcription from the opposite direction; (3) promoter 3, about $35 \mathrm{~kb}$ upstream of Gs $\alpha$ exon 1, encodes extra-large alphas protein (XL $\alpha s$ ),

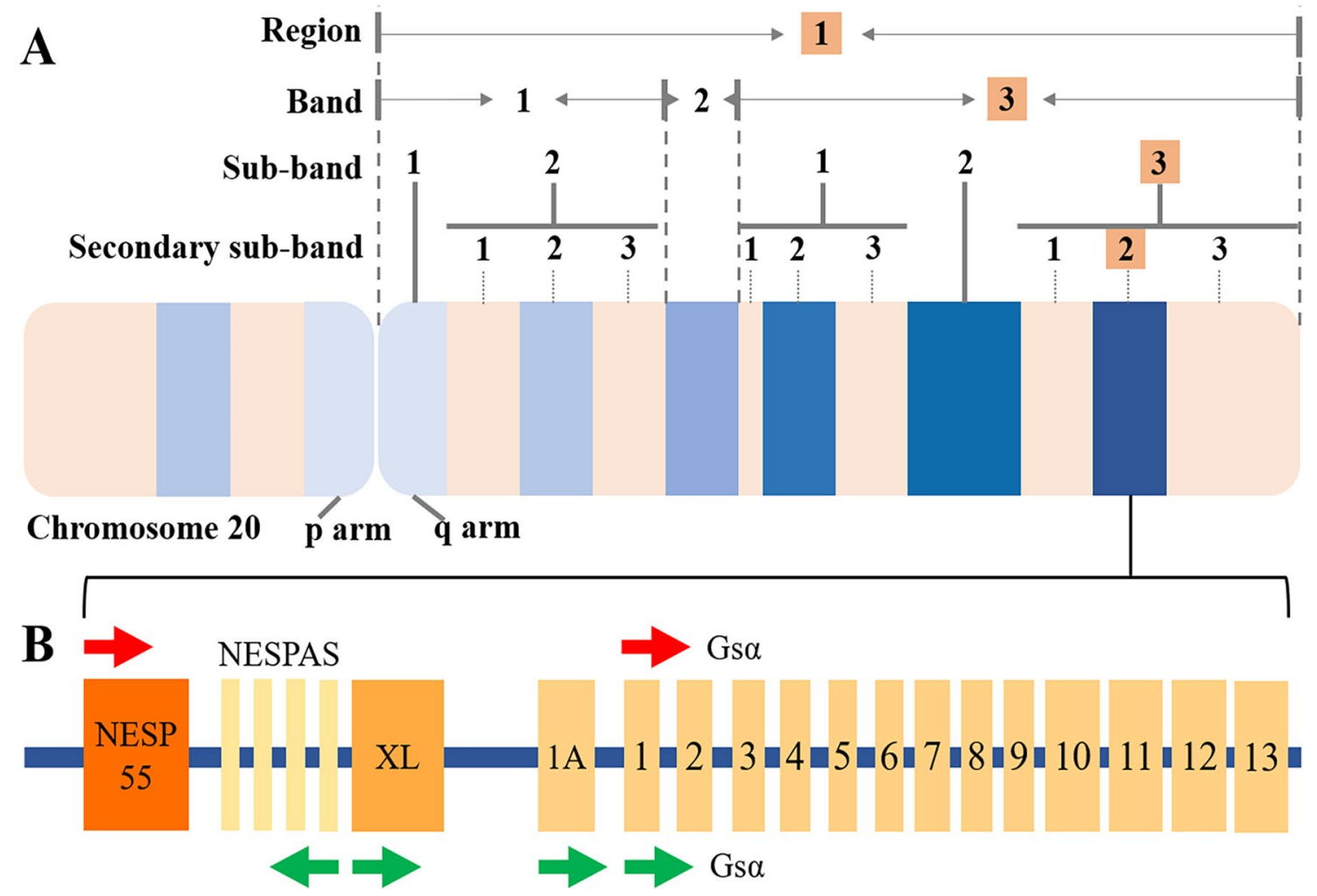

Maternal expression $\rightarrow$ Paternal expression

Fig. 1 The location and biological structure of GNAS gene. a GNAS gene is located at chromosome 20q13.32; b thirteen exons and the upstream alternative first exons of GNAS. The imprinted expression pattern of GNAS is highly complicated, with exclusively maternal expression of NESP55 (red arrow), and exclusively paternal expression of NESPAS, XL exon and exon 1A (green arrow). NESPAS: NESP anti sense 
whose coding sequence is composed of XL exon and Gs $\alpha$ exon 1; (4) promoter 4 locates at about $2.5 \mathrm{~kb}$ upstream of Gs $\alpha$ exon 1. The resulted exon 1A transcripts were presumed to be untranslated mRNAs. The imprinted expression patterns of the aforementioned promoters are highly complicated. NESP55 is maternally expressed, while NESP anti sense, XL $\alpha$ s, and exon $1 \mathrm{~A}$ are paternally expressed (Fig. 1b) (Crane et al. 2009).

The UniProt database (https://www.uniprot.org/) was used to search for proteins encoded by GNAS, with the searching term as "gene: GNAS AND reviewed: yes AND organism: "Homo sapiens (Human) [9606]"”. The result showed four kinds of proteins encoded by GNAS: (1) Gs $\alpha$, with a length of 394 amino acid residues, is encoded by GNAS exon 1-13; (2) XLas, with a length of 1037 amino acid residues, is paternally expressed and responsible for the stimulation of AC-cAMP-PKA signaling pathway. XL $\alpha \mathrm{s}$ is one of the isoforms of Gs $\alpha$, with similar downstream receptor to Gs $\alpha$. But there is no evidence showing that seventransmembrane receptors activating Gs $\alpha$ can also activate XLas; (3) protein ALEX, with a length of 626 amino acid residues, is the product of paternal expression of XL exon and possibly contributes to the inhibition of AC activity in XLos subunit (Abramowitz et al. 2004); (4) NESP55, with a length of 245 amino acids, is maternally expressed and encoded by NESP55 exon. NESP55 forms LHAL tetrapeptide and GPIPIRRH peptide after modification and shear.

\section{The structure and function of Gsa}

Among the four reviewed proteins, Gs $\alpha$ is the main product of GNAS gene, which includes two domains (Rose et al. 2018) (Fig. 2). The first is guanosine triphosphate enzyme (GTPase) domain, which is formed after the fold of 39-394th amino acid residues. GTPase domain functions as the guanosine-biding and interaction site for receptors and effectors. There are four guanosine triphosphate/guanosine diphosphate (GTP/GDP)-binding sites, located at 47-55th, 197-204th, 223-227th, and 292-295th amino acid residues respectively; and two magnesium ion-binding sites, located at 54th and 204th amino acid residues, respectively. Two out of the four GTP/GDP-binding sites are highly conserved [arginine ${ }^{201}\left(\operatorname{Arg}^{201}\right)$ and glutamine ${ }^{227}\left(\mathrm{Gln}^{227}\right)$ ], which play a vital role on the hydrolysis of the bound GTP. The second is helical domain, with a possible function of maintaining the binding status between GTP/GDP and Gs $\alpha$ (Weinstein et al. 2001). Besides the four domains, there are five motif structures in Gs $\alpha$, including G1 (42-55th amino acids), G2 (196-204th amino acids), G3 (219-228th amino acids), G4 (288-295th amino acids), and G5 (364-369th amino acids).

The signaling from GPCR to the downstream molecules is carried out through G protein cycle (Fig. 3, red-dotted box): (1) Gs $\alpha$ releases GDP and combines with GTP due to the affinity reduction between Gs $\alpha$ and GDP caused by activation from ligand-binding GPCR to Gs $\alpha$; (2) GTPbinding Gs $\alpha$ separates with $\beta$ and $\gamma$ subunits and turns into an activated status, which is able to stimulate downstream molecules; (3) as reacting with the downstream molecules,
Fig. 2 The tertiary structure of Gs $\alpha$ subunit (cited from RCSB PDB database, https://www. rcsb.org/; PDB ID: 6AU6). Magenta and purple: $\alpha$-helix; yellow and blue: $\beta$-sheet; white: random coil. Arg arginine, Gln glutamine, GTP guanosine triphosphate, GTPase GTP enzyme, GDP guanosine diphosphate

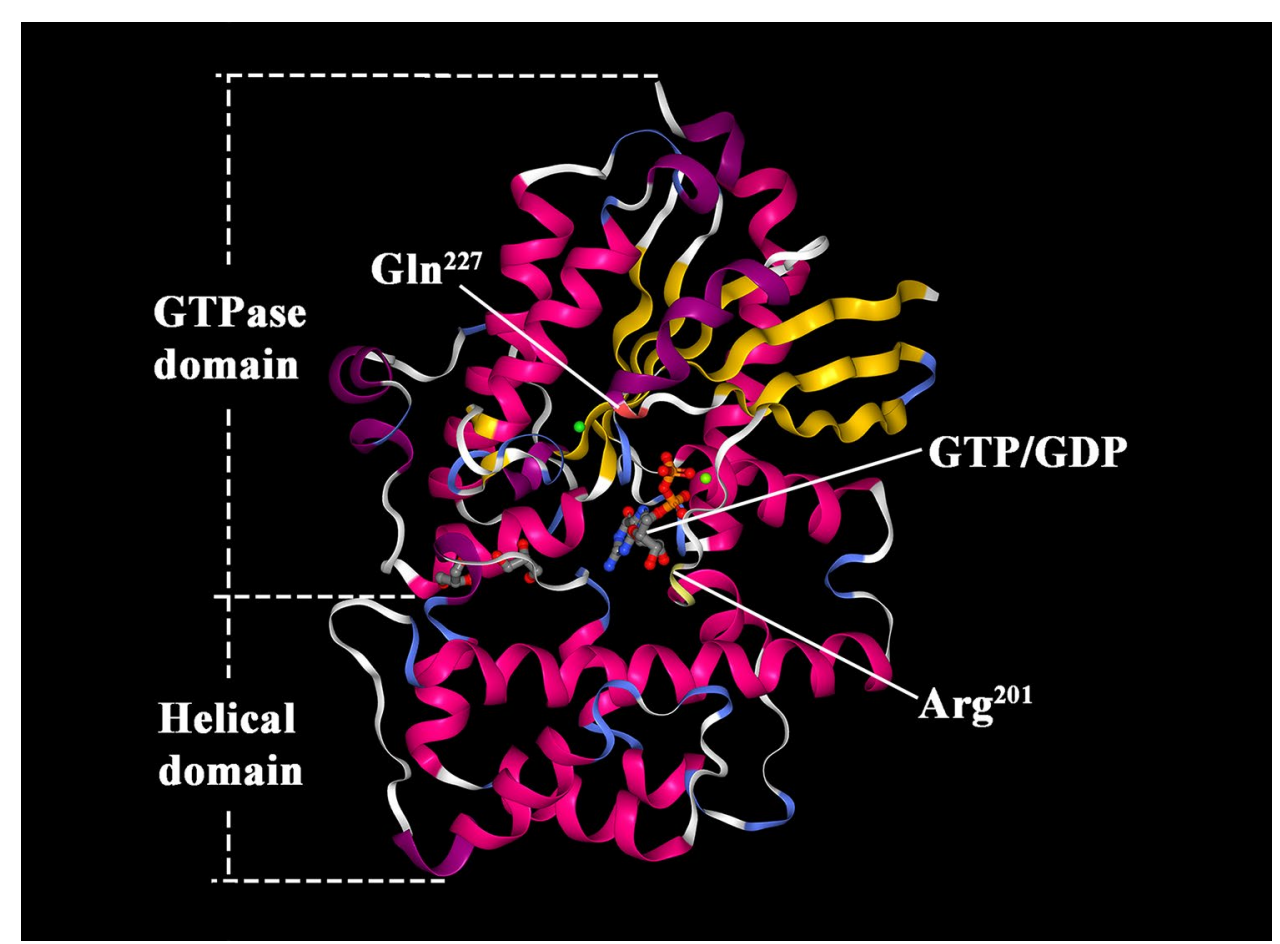




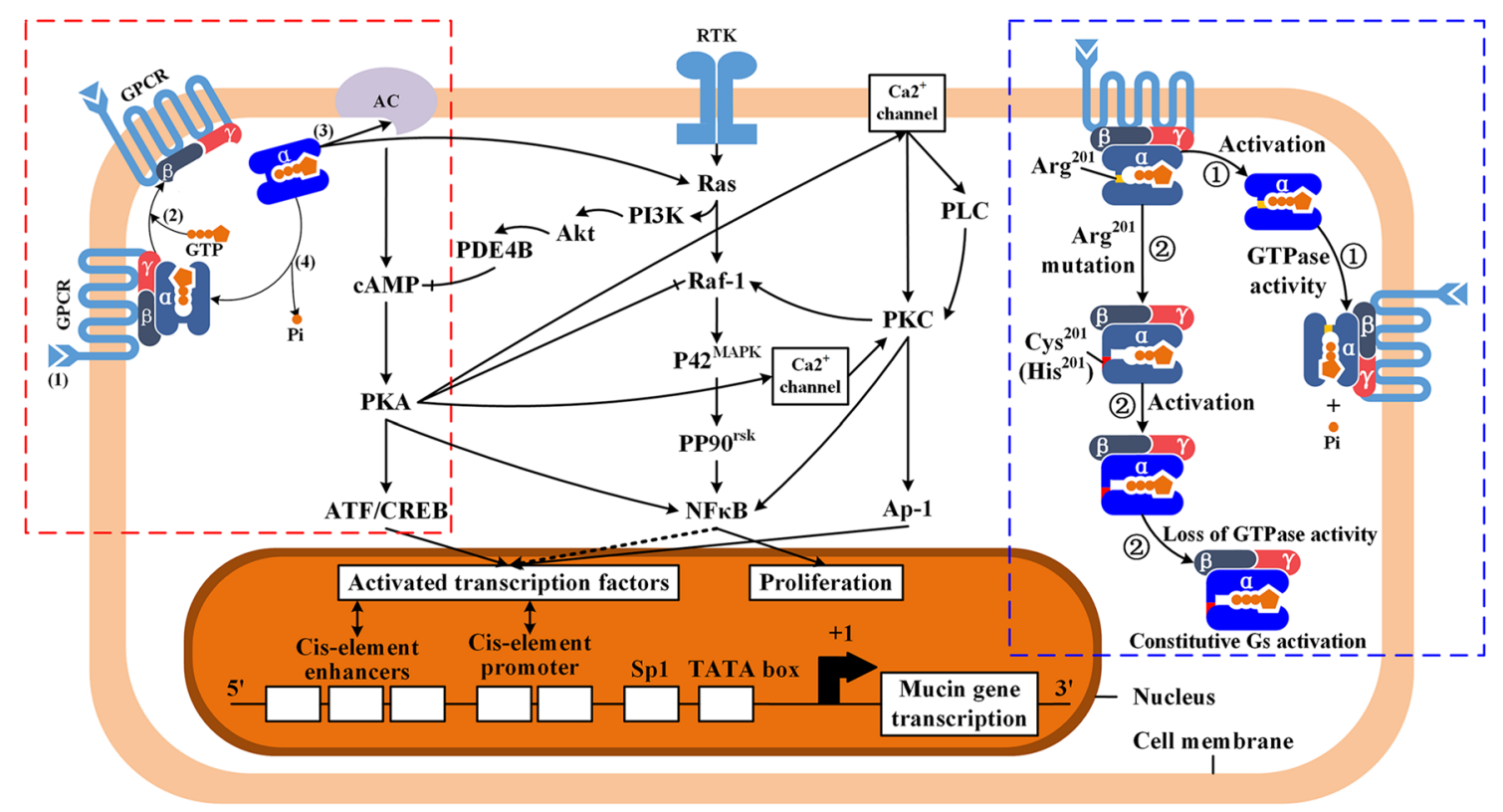

Fig. $3 \mathrm{G}$ protein cycle, activity changes of Gs $\alpha$ subunit caused by GNAS mutation, and the crosslink among Gs $\alpha$ subunit-induced cAMP-PKA, PI3K-Akt, and RAS-MAPK pathways. Red dotted box: $\mathrm{G}$ protein cycle; blue dotted box: activity changes of Gs $\alpha$ subunit caused by the mutation of Gs $\alpha \mathrm{Arg}^{201}$. (1) In the physiological status, activated Gs $\alpha$ returns to deactivated status after releasing a Pi; (2) In the situation of $\mathrm{Arg}^{201}$ mutation, Gs $\alpha$ fails to release Pi and remains in activated status. Gs $\alpha$ in ink blue: deactivated status. Gs $\alpha$ in light blue: activated status; the other signaling pathways: cAMPPKA, PI3K-Akt, and RAS-MAPK pathways interacts among each other and eventually modulate mucin gene expression via the nuclear import of ATF/CREB and NFkB. GPCR G protein-coupled recep-

the GTPase activity of Gs $\alpha$ is activated and then GTP is hydrolyzed. Eventually, Gs $\alpha$ returns to the primary structure and reforms trimer with $\beta$ and $\gamma$ subunits.

\section{The molecular changes of GNAS mutation}

A thorough literature research identified 13 papers reporting the genetic variants and corresponding gene mutation rates in PMP. Only variants reported in $\geq 5$ papers were listed in Table 1. As listed in Table 1, the two most frequent variants in PMP are KRAS and GNAS mutations, with a median mutation rates of $77.8 \%$ (range $40.0-100 \%$ ) and $45.7 \%$ (range $25.7-100 \%$ ) respectively. By reviewing papers describing the detailed variant form of GNAS, we found that the most frequently detected GNAS mutation forms were c.602G >A (p.R201H) and c.601C > T (p.R201C) (Table 2). Despite the different variant forms reported by Pengelly et al. (2018) and Saarinen et al. (2017), the variant sites were relatively stable, both located at Chr20: 57,484,420 and Chr20: 57,484,421, which was identical to c.602G $>$ A (p.R201H) and c.601C $>$ T (p.R201C). Various transcripts tor, $G s \alpha$ stimulatory $\mathrm{G}$ protein subunit, $P i$ inorganic phosphate, $A C$ adenyl cyclase, $c A M P$ cyclic adenosine monophosphate, $P K A$ protein kinase A, $A T F$ activating transcription factor, $C R E B$ cAMP-response element-binding protein, $P L C$ phospholipase $\mathrm{C}, P K C$ protein kinase C, $P I 3 K$ phosphoinositide 3-kinase, Akt protein kinase B, PDE4B phosphodiesterase $4 \mathrm{~B}, R T K$ receptor tyrosine kinases, Ras rat sarcoma protein, Raf-1 Raf-1 protein, $P 42^{M A P K}$ P42 mitogen-activated protein kinas, also named Erk2, extracellular signal-regulated kinase 2, $P P 90^{r s k} 90 \mathrm{kDa}$ ribosomal S6 kinase, $N F \kappa B$ nuclear factor kappalight-chain-enhancer of activated B cells, $S p 1$ specificity protein 1, Arg arginine, Cys cysteine, His histidine

chosen after sequencing might have resulted in the different expression patterns of mutation sites. Thus, it can be concluded that Chr20: 57,484,420 C>T (c.601C > T: p.R201C) and Chr20: 57,484,421 G>A (c.602G >A: p.R201H) are the two most significant variant forms in PMP GNAS mutations.

Taking the encoding of Gs $\alpha$ for example, once c.601C $>\mathrm{T}$ and c.602G $>$ A mutation occur, the 201th amino acid residue, Arg, changes into cysteine (Cys) and histidine (His) respectively. The variants significantly alter the structure of GTPase domain in Gs $\alpha$, and vastly decrease GTPase activity. As a consequence, Gs $\alpha$ fails to hydrolyze GTP and release phosphoric acid, remaining in activated status, which continuously stimulates downstream molecules (Fig. 3, blue dotted box).

\section{Influences of GNAS mutation to mucin secretion and cell proliferation}

\section{Mucin expression in PMP}

There are two major types of mucins, gel-forming mucins and transmembrane mucins (Johansson and Hansson 2016). 
Table 1 Summary of the top 5 mutations in pseudomyxoma peritonei

\begin{tabular}{|c|c|c|c|c|c|c|c|c|c|}
\hline \multirow{2}{*}{ References } & \multirow{2}{*}{ Cases } & \multirow[t]{2}{*}{ Gene panel } & \multicolumn{7}{|c|}{ Gene mutation rates $(\%)$} \\
\hline & & & $K R A S$ & GNAS & $K R A S+G N A S$ & TP53 & $S M A D 4$ & $A P C$ & $P I 3 C A$ \\
\hline Tokunaga et al. (2019) & 183 & 592 & 55.0 & 31.0 & NA & 40.0 & 16.0 & 10.0 & 6.0 \\
\hline Pengelly et al. (2018) & 5 & 54 & 100.0 & 100.0 & 100.0 & NA & 10.0 & 10.0 & NA \\
\hline Gleeson et al. (2018) & $19-31^{\mathrm{a}}$ & 47 & 80.6 & 73.7 & 87.0 & 5.0 & 16.0 & 11.0 & 10.0 \\
\hline Saarinen et al. (2017) & 9 & Whole exome & 100.0 & 55.6 & 55.6 & NA & NA & NA & NA \\
\hline Borazanci et al. (2017) & $116-396^{\mathrm{a}, \mathrm{b}}$ & 47 & 57.3 & 28.2 & NA & 23.4 & 16.2 & 10.7 & 5.3 \\
\hline Pietrantonio et al. (2016a, b) & 40 & 50 & 72.0 & 52.5 & NA & 12.5 & 2.5 & NA & 7.5 \\
\hline Nummela et al. (2015) & 19 & 48 & 100.0 & 63.2 & NA & 5.3 & 15.3 & 0.0 & 5.3 \\
\hline Noguchi et al. (2015) & 18 & 50 & 77.8 & 44.4 & NA & 22.2 & 16.7 & NA & 11.1 \\
\hline Sio et al. (2014) & 10 & 236 & 70.0 & 40.0 & 40.0 & NA & NA & NA & NA \\
\hline Liu et al. (2014) & 35 & 50 & 42.9 & 25.7 & NA & 20.0 & 14.3 & 22.9 & 5.7 \\
\hline Alakus et al. (2014) & 29 & NA & 89.7 & 70.0 & NA & 0.0 & NA & NA & 0.0 \\
\hline Singhi et al. (2014) & 55 & 2 & 40.0 & 31.0 & NA & NA & NA & NA & NA \\
\hline Nishikawa et al. (2013) & 35 & 2 & 94.3 & 45.7 & 42.9 & NA & NA & NA & NA \\
\hline Range & NA & NA & $40.0-100.0$ & $25.7-100.0$ & $40.0-100.0$ & $0-40.0$ & $2.5-16.7$ & $0-22.9$ & $0-11.1$ \\
\hline Median & NA & NA & 77.8 & 45.7 & 55.6 & 16.3 & 15.7 & 10.4 & 5.9 \\
\hline
\end{tabular}

NA not available

${ }^{\mathrm{a}}$ Number of patients varied by different genes detected

${ }^{\mathrm{b}}$ Patients with neuroendocrine tumors of appendix were excluded

Gel-forming mucins mainly include MUC2, MUC5AC, MUC5B, and MUC6. Transmembrane mucins mainly consist of MUC1, MUC3, MUC4, MUC12, MUC13, MUC16, and MUC17. A thorough review of the published literatures on mucin expression in PMP identified some distinctive features (Table 3). First, most researches focus on the expression status of gel-forming mucins, while little attention has been paid to transmembrane mucins. Second, MUC2 and MUC5AC are the most frequently expressed gel-forming mucins in PMP, with positive rates being 99.1\% (314/317) and $96.5 \%$ (193/200), respectively, among the detected samples. MUC6 is rarely detected in PMP compared with MUC2 and MUC5AC, with positive rate of $12.5 \%$ (2/16). Third, the transmembrane MUC1 expresses variably in PMP, with positive rate being $41.3 \%$ (33/80). The expression status of MUC4 is currently unclear due to the limitation of sample number. Based on the available data from published literatures, it is advisable to focus more attention on in-depth study on MUC2 and MUC5AC.

\section{GNAS functions on the regulation of mucin secretion}

GNAS mutation is frequently detected in mucinous neoplasms of appendix (50\%) and intraductal papillary mucinous neoplasm (IPMN) of pancreas (81\%) (Furukawa et al. 2011; Wu et al. 2011), while the mutation rate in mucinous adenocarcinoma of colorectum, ovary, lung, and breast are relatively lower, even being 0\% (Nishikawa et al. 2013). In addition, both PMP and IPMN share similar inertia biological behavior as well as hypersecretion of mucus. Therefore, it is inferred that GNAS might play some role in the regulation of mucin secretion (Alakus et al. 2014; Noguchi et al. 2015; Tokunaga et al. 2019).

The effect of GNAS mutation to mucin secretion has been proved by Nishikawa et al. (2013). The author transfected HT29 cells with an EF1a-GNAS ${ }^{\text {R201H }}$-IRES-Zeo plasmid. The result showed that cAMP, MUC2, and MUC5AC level elevated after the expression of GNAS ${ }^{\mathrm{R} 201 \mathrm{H}}$. While the application of PKA inhibitor downregulated the expression of $M U C 2$ and $M U C 5 A C$ genes. Nishikawa's study demonstrates that GNAS mutation might regulate mucin production through cAMP-PKA signaling pathway (Bradbury 2000; Jarry et al. 1994). The potential regulation method of cAMP-PKA signaling pathway might be stimulating cAMP-response element-binding protein (CREB) and activating transcription factor (ATF) family (Velcich and Augenlicht 1993). After entering nucleus, the activated CREB/ATF combines to the upstream cis-acting element of mucin genes and thus regulate mucin expression. Other studies have also proved that inhibitors of both PKA and heterotrimer $\mathrm{G}$ protein complex could also significantly downregulate mucin expression. Although GNAS mutation is proved to be an important promoter in mucin secretion of PMP, the current experiment was performed in colorectal cancer cell lines due to the difficulties in the culture of PMP tumor cells (Nishikawa et al. 2013). Besides, the influence of GNAS mutation to different types of mucin still needs further exploration. 


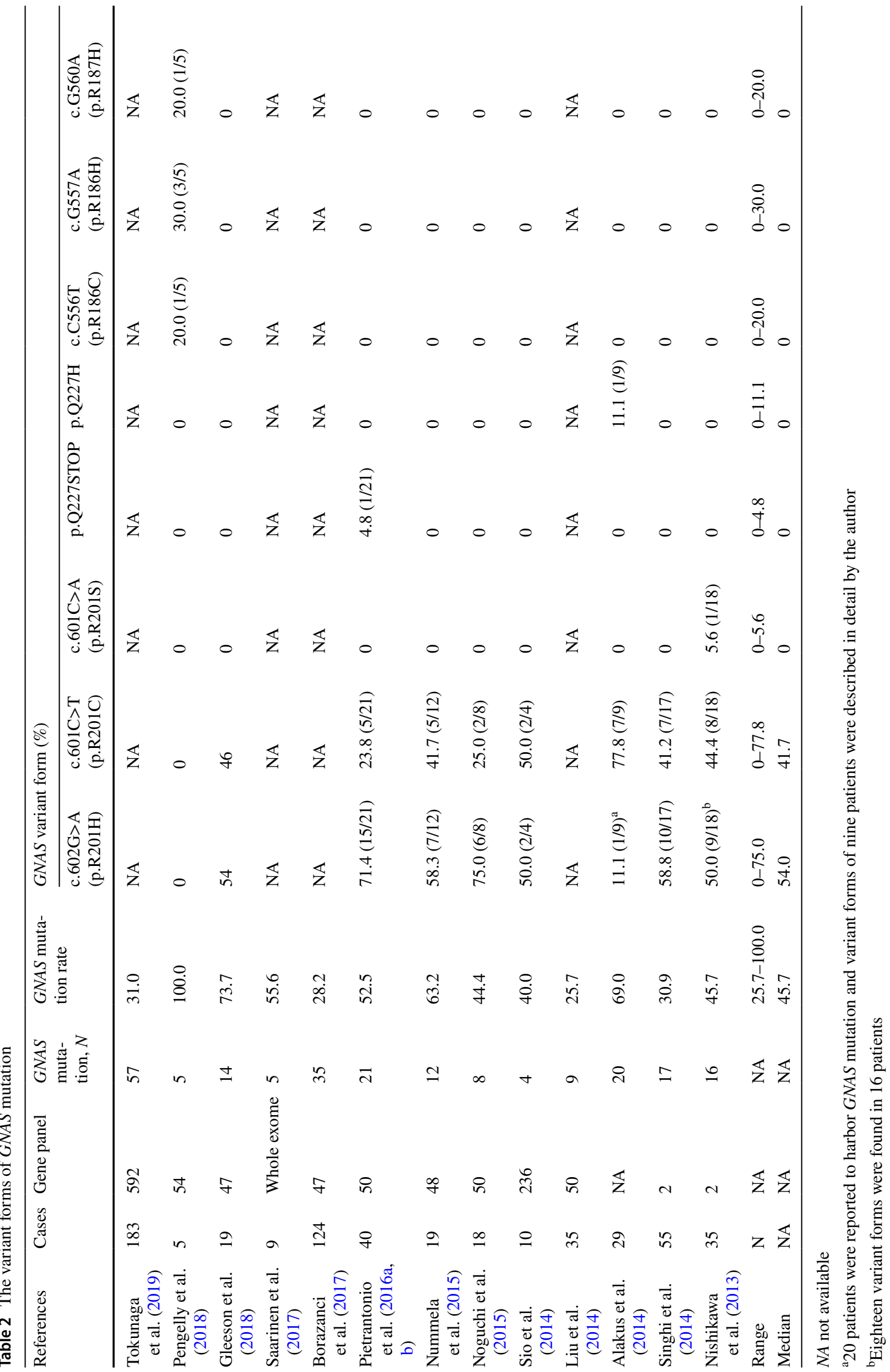


Table 3 Mucin expression status in pseudomyxoma peritonei

\begin{tabular}{|c|c|c|c|c|c|c|c|}
\hline \multirow[t]{2}{*}{ References } & \multirow[t]{2}{*}{ Cases } & \multirow[t]{2}{*}{ Method } & \multicolumn{3}{|c|}{ Gel-forming mucins (\%) } & \multicolumn{2}{|c|}{$\begin{array}{l}\text { Transmembrane } \\
\text { mucins (\%) }\end{array}$} \\
\hline & & & MUC2 & MUC5AC & MUC6 & MUC1 & MUC4 \\
\hline Yan et al. (2019) & 21 & IHC & 100 & 100 & NA & NA & NA \\
\hline Yan et al. (2020) & 5 & IHC & 100 & NA & NA & 60 & NA \\
\hline Li et al. $(2017 a, b)$ & 9 & IHC & 100 & NA & NA & NA & NA \\
\hline Li et al. $(2017 a, b)$ & 8 & IHC & 100 & NA & NA & NA & NA \\
\hline Guo et al. (2011) & 35 & $\mathrm{IHC}$ & 97.1 & NA & NA & 0 & NA \\
\hline Flatmark et al. (2010) & 5 & $\mathrm{IHC}$ & 100 & 60.0 & NA & 0 & 100 \\
\hline Ferreira et al. (2008) & 7 & $\mathrm{IHC}$ & 100 & 100 & 28.6 & 28.6 & NA \\
\hline Semino-Mora et al. (2008) & 16 & FISH & 100 & 100 & NA & NA & NA \\
\hline McKenney and Longacre (2008) & 1 & $\mathrm{IHC}$ & 100 & NA & NA & NA & NA \\
\hline Nonaka et al. (2006) & 42 & $\mathrm{IHC}$ & 100 & 100 & NA & NA & NA \\
\hline Heiskala et al. (2006) & 9 & IHC & 100 & 100 & 0 & NA & NA \\
\hline Bibi et al. (2006) & 26 & $\mathrm{IHC}$ & 100 & NA & NA & NA & NA \\
\hline Mohamed et al. (2004) & 33 & IHC & 100 & NA & NA & 84.8 & NA \\
\hline O'Connell et al. (2002a, b) & 100 & $\mathrm{IHC}$ & 98.0 & 95 & NA & NA & NA \\
\hline Total & 317 & NA & 99.1 & 96.5 & 12.5 & 41.3 & 100 \\
\hline Range & NA & NA & $97.1-100$ & $60.0-100$ & $0-28.6$ & $0-84.8$ & $100-100$ \\
\hline Median & NA & NA & 100 & 100 & 14.3 & 28.6 & 100 \\
\hline
\end{tabular}

MUC2 mucin 2, MUC5AC mucin 5AC, IHC immunohistochemistry, FISH fluorescence in situ hybridization, $N A$ not available
The existed pathways which have cross reaction with cAMP-PKA pathway also participate in the regulation of mucin expression indirectly (Fig. 3): (1) MAPK signaling pathway. The activated cAMP influences MAPK signaling pathway via activating Ras or inhibiting Raf- 1 by PKA. In pulmonary cystic fibrosis, it has been illustrated that hyperexpression of MUC2 was mainly regulated through $\mathrm{Src} / \mathrm{Ras} / \mathrm{MAPK} / \mathrm{pp} 90^{\text {rsk }}$ signaling pathway (Li et al. 1998). However, the function of Src/Ras/MAPK/pp90 rsk in PMP is not proven currently; (2) Ras-PI3K-Akt signaling pathway. PDE4B activated by this pathway functions as an antagonist against cAMP-PKA signaling pathway by clearing cAMP (Alakus et al. 2014); (3) PKC signaling pathway. Activated PKC has synergistic effect on cAMP-PKA pathway through activating Raf-1. Besides, $\mathrm{Ca}^{2+}$-dependent PKC-epsilon could also upregulate $M U C 2$ and MUC5AC expression (Hong et al. 1999).

\section{GNAS functions on the regulation of tumor cell proliferation}

Generally, the current studies support the notion that PMP and colorectal cancer share similar gene mutation profiles, but vary vastly in mutation rate. PMP possesses higher mutation rates in GNAS and KRAS, while lower mutation rates in TP53, APC, and PIK3CA (Alakus et al.
2014; Tokunaga et al. 2019). Nishikawa et al. transfected HT29 cells with an EF1a-GNAS ${ }^{\text {R201H }}$-IRES-Zeo plasmid. The cell proliferation remained the same, but accompanied with elevated mucin secretion. The result indicated that GNAS mutation mainly affect the expression level of mucin instead of tumor cell proliferation. KRAS is another important variant in PMP, and has been reported to promote tumor cell proliferation through the activation of MAPK signaling pathway (Alakus et al. 2014; PylayevaGupta et al. 2011).

\section{Impacts of GNAS mutation to clinical- pathological characteristics and prognosis}

\section{Correlation of GNAS mutation and clinical- pathological characteristics}

In a study cohort of 55 patients, Singhi et al. (2014) demonstrated no significant association between GNAS mutation and gender, age, and adverse histological features (including cytologic grade, destructive invasion, tumor cellularity, angiolymphatic invasion, perineural invasion, and signet ring cells $)(P>0.05)$. However, the author found that GNAS-mutated PMP was prone to harbor concurrent KRAS mutation compared with GNAS-wild-type PMP $(65 \%$ vs. $29 \%, P=0.018)$. 
Pietrantonio et al. (2016a, b) analyzed 15 patients with relapsed PMP, and revealed no association between GNAS mutation and gender, age, Eastern Cooperative Oncology Group performance status, histological grade, time elapsed from surgery to relapse, peritoneal cancer index (PCI), and completeness of cytoreduction. In another study of 40 PMP patients, Pietrantonio et al. (2016a, b) found that GNAS mutation was correlated to incomplete cytoreduction $(P=0.05)$ and KRAS mutation $(P=0.002)$. Besides, neither GNAS nor KRAS mutation were associated with pathological grade $(P=0.338$ and 0.427 , respectively).

From the studies by Pietrantonio et al. (2016a, b) and Singhi et al. (2014), it could be inferred that the presence of GNAS mutation is related to KRAS mutation. Considering the high incidence of these two variants in PMP and the statistically close relationship, the independent and synergistic effect as wells as the crosslink between GNAS and $K R A S$ could be important issues to be explored in the mechanical studies of PMP.

Despite of the application of different criteria in histopathological classification, most of the studies showed that GNAS mutational status had no association with histopathological grade (Gleeson et al. 2018; Nummela et al. 2015; Pietrantonio et al. 2016a, b; Singhi et al. 2014). However, opposite opinions existed concerning the relation between GNAS mutation and histopathological grade. Noguchi et al. (2015) investigated mutation profiles of 18 PMP patients, revealing GNAS mutation in five low-grade PMP and three high-grade PMP. Noguchi hold the view that GNAS mutation might play a key role in both lowgrade and high-grade PMP. On the contrast, in a study performed by Alakus et al. (Alakus et al. 2014), the result revealed that GNAS mutation rate is lower in high-grade PMP (21/23 vs. $1 / 6, P=0.005)$. For the only patient with high-grade PMP presenting GNAS mutation, it was observed that the histopathology of the intraperitoneal implantation was a mixture of partly low-grade and partly high-grade PMP. Considering the existence of low-grade loci, Alakus et al. made a conclusion that high-grade PMP might not evolve from low-grade PMP.

\section{Impacts of GNAS mutation on PMP prognosis}

Few studies were performed to investigate the association between GNAS mutation and prognosis of PMP. The results varied among different studies. Singhi et al. (2014) found that GNAS mutation did not affect the overall survival (OS) or time to disease progression. High tumor grade (AJCC G2 and G3) $(P=0.002)$ and lymph node involvement $(P=0.025)$ were associated with poorer OS. While HIPEC was associated with improved OS. Cox proportional hazard model identified that only lymph node involvement was the independent prognostic factor of PMP. In a study performed by Pietrantonio et al. (2016a, b), it was found that patients with GNAS mutation had significantly shorter median progression-free survival (PFS) than GNAS-wild type patients (5.3 months vs. not reached, $P<0.007$ ). Later, in a study cohort of 40 patients, Pietrantonio et al. again demonstrated that GNAS mutation was associated with PFS. The other variables correlated to PFS were completeness of cytoreduction score, PCI score, and KRAS mutation status. However, multiple variate analysis revealed only PCI $>20$ and KRAS mutation were the independent predictors of PFS.

\section{Summary}

To sum up, GNAS mutation is one of the most important molecular biological features in PMP, which might function as promoting the secretion of mucin. The mutation sites of GNAS mutation is relatively stable, usually at Chr20: 57,484,420 (base pair: C-G) and Chr20: 57,484,421 (base pair: G-C). The presence of GNAS mutation results in the reduction of GTPase activity in Gs $\alpha$, causing failure to hydrolyze GTP and release phosphoric acid, and eventually the continuous combining status of Gs $\alpha$ and GTP. The activated Gs $\alpha$ could thus continuously stimulate mucin secretion through the stimulation of cAMP-PKA signaling pathway. As presented above, there were already several studies proving that GNAS could elevate secretion level of mucin, but the experiments were limited in the cell lines of colorectal cancer. A more reliable evidence provided by experiments of genetic and protein level in PMP cell line is in urgent requirement.

The high mutation rate of GNAS in PMP patients has been observed about 10 years ago, when fresh tumor tissue or formalin-fixed, paraffin-embedded tissue was used for variant detection. However, the number of patients was limited, and most of the sequencings were non-whole-exome sequencing, which indicated the deficiency on the comprehensive view of PMP mutation profile. Generally speaking, the establishment of stable PMP cell line combined with comprehensive mutation profile would vastly help to improve the understanding of PMP genetically, and uncover the mechanism of PMP, especially the influence of GNAS mutation to mucin hypersecretion, which might eventually facilitate the innovation of new drugs targeting the molecules in the GNAS-related signaling pathways.

Acknowledgements Beijing Municipal Administration of Hospitals' Ascent Plan (DFL20180701); Special Fund for the Capital Characteristic Clinical Medicine Development Project (Z161100000516077); Beijing Municipal Grant for Medical Talents Group on Peritoneal Surface Oncology (2017400003235J007); Key Discipline Development Fund of Beijing Shijitan Hospital, Capital Medical University (2016fmzlwk); Beijing Natural Science Foundation (7172108); Beijing Health and 
Science Technology Achievement and Appropriate Technology Promotion Project (2018-TG-27).

Author contributions Y-LL was responsible for literature review, data collection and analysis, summary, and manuscript writing; RM helped to summarize tables and revise/review manuscript; YL designed the study and refined manuscript definition of intellectual content. Manuscript of final version was approved by YL.

Funding This study was supported by funding from Beijing Municipal Administration of Hospitals' Ascent Plan (DFL20180701); Special Fund for the Capital Characteristic Clinical Medicine Development Project, Beijing Municipal Science and Technology Commission (Z161100000516077); Beijing Municipal Grant for Medical Talents Group on Peritoneal Surface Oncology (2017400003235J007); Key Discipline Development Fund of Beijing Shijitan Hospital, Capital Medical University (2016fmzlwk); Beijing Natural Science Foundation (7172108); Beijing Health and Science Technology Achievement and Appropriate Technology Promotion Project (2018-TG-27).

Availability of data and material All data and material generated or used during the study are available from the corresponding author by request.

\section{Compliance with ethical standards}

Conflict of interest The authors declare that they have no competing interests.

Open Access This article is licensed under a Creative Commons Attribution 4.0 International License, which permits use, sharing, adaptation, distribution and reproduction in any medium or format, as long as you give appropriate credit to the original author(s) and the source, provide a link to the Creative Commons licence, and indicate if changes were made. The images or other third party material in this article are included in the article's Creative Commons licence, unless indicated otherwise in a credit line to the material. If material is not included in the article's Creative Commons licence and your intended use is not permitted by statutory regulation or exceeds the permitted use, you will need to obtain permission directly from the copyright holder. To view a copy of this licence, visit http://creativecommons.org/licenses/by/4.0/.

\section{References}

Abramowitz J, Grenet D, Birnbaumer M, Torres HN, Birnbaumer L (2004) XLalphas, the extra-long form of the alpha-subunit of the Gs $\mathrm{G}$ protein, is significantly longer than suspected, and so is its companion Alex. Proc Natl Acad Sci USA 101:8366-8371

Alakus H et al (2014) Genome-wide mutational landscape of mucinous carcinomatosis peritonei of appendiceal origin. Genome Med 6:43. https://doi.org/10.1186/gm559

Bibi R, Pranesh N, Saunders MP, Wilson MS, O’Dwyer ST, Stern PL, Renehan AG (2006) A specific cadherin phenotype may characterise the disseminating yet non-metastatic behaviour of pseudomyxoma peritonei. Br J Cancer 95:1258-1264. https://doi. org/10.1038/sj.bjc.6603398

Bird AP (1986) CpG-rich islands and the function of DNA methylation. Nature 321:209-213. https://doi.org/10.1038/321209a0

Borazanci E, Millis SZ, Kimbrough J, Doll N, Von Hoff D, Ramanathan RK (2017) Potential actionable targets in appendiceal cancer detected by immunohistochemistry, fluorescent in situ hybridization, and mutational analysis. J Gastrointest Oncol 8:164-172. https://doi.org/10.21037/jgo.2017.01.14

Bradbury NA (2000) Protein kinase-A-mediated secretion of mucin from human colonic epithelial cells. J Cell Physiol 185:408-415. https://doi.org/10.1002/1097-4652(200012)185:3<408:AIDJCP11>3.0.CO;2-2

Chua TC et al (2012) Early- and long-term outcome data of patients with pseudomyxoma peritonei from appendiceal origin treated by a strategy of cytoreductive surgery and hyperthermic intraperitoneal chemotherapy. J Clin Oncol 30:2449-2456. https://doi. org/10.1200/JCO.2011.39.7166

Crane JL, Shamblott MJ, Axelman J, Hsu S, Levine MA, Germain-Lee EL (2009) Imprinting status of Galpha(s), NESP55, and XLalphas in cell cultures derived from human embryonic germ cells: GNAS imprinting in human embryonic germ cells. Clin Transl Sci 2:355-360. https://doi.org/10.1111/j.1752-8062.2009.00148.x

Ferreira CR, Carvalho JP, Soares FA, Siqueira SAC, Carvalho FM (2008) Mucinous ovarian tumors associated with pseudomyxoma peritonei of adenomucinosis type: immunohistochemical evidence that they are secondary tumors. Int J Gynecol Cancer 18:59-65. https://doi.org/10.1111/j.1525-1438.2007.00988.x

Flatmark K, Davidson B, Kristian A, Stavnes HT, Forsund M, Reed W (2010) Exploring the peritoneal surface malignancy phenotype-a pilot immunohistochemical study of human pseudomyxoma peritonei and derived animal models. Hum Pathol 41:1109-1119. https://doi.org/10.1016/j.humpath.2009.12.013

Furukawa T et al (2011) Whole-exome sequencing uncovers frequent GNAS mutations in intraductal papillary mucinous neoplasms of the pancreas. Sci Rep 1:161. https://doi.org/10.1038/srep00161

Gardiner-Garden M, Frommer M (1987) CpG islands in vertebrate genomes. J Mol Biol 196:261-282. https://doi.org/10.1016/00222836(87)90689-9

Germain-Lee EL et al (2005) A mouse model of albright hereditary osteodystrophy generated by targeted disruption of exon 1 of the Gnas gene. Endocrinology 146:4697-4709. https://doi. org/10.1210/en.2005-0681

Gleeson EM et al (2018) Appendix-derived pseudomyxoma peritonei (PMP): molecular profiling toward treatment of a rare malignancy. Am J Clin Oncol 41:777-783. https://doi.org/10.1097/coc.00000 00000000376

Guo AT, Song X, Wei LX, Zhao P (2011) Histological origin of pseudomyxoma peritonei in Chinese women: clinicopathology and immunohistochemistry. World J Gastroenterol 17:3531-3537. https://doi.org/10.3748/wjg.v17.i30.3531

Heiskala K, Giles-Komar J, Heiskala M, Andersson LC (2006) High expression of RELP (Reg IV) in neoplastic goblet cells of appendiceal mucinous cystadenoma and pseudomyxoma peritonei. Virchows Arch 448:295-300. https://doi.org/10.1007/s0042 8-005-0105-1

Hong DH, Petrovics G, Anderson WB, Forstner J, Forstner G (1999) Induction of mucin gene expression in human colonic cell lines by PMA is dependent on PKC-epsilon. Am J Physiol 277:G10411047. https://doi.org/10.1152/ajpgi.1999.277.5.G1041

Jarry A, Merlin D, Hopfer U, Laboisse CL (1994) Cyclic AMP-induced mucin exocytosis is independent of Cl-movements in human colonic epithelial cells (HT29-Cl.16E). Biochem J 304:675-678

Johansson ME, Hansson GC (2016) Immunological aspects of intestinal mucus and mucins. Nat Rev Immunol 16:639-649. https:// doi.org/10.1038/nri.2016.88

Li JD, Feng W, Gallup M, Kim JH, Gum J, Kim Y, Basbaum C (1998) Activation of NF-kappaB via a Src-dependent Ras-MAPKpp90rsk pathway is required for Pseudomonas aeruginosainduced mucin overproduction in epithelial cells. Proc Natl Acad Sci USA 95:5718-5723 
Li Y, Yu Y, Liu Y (2014) Report on the 9(th) international congress on peritoneal surface malignancies. Cancer Biol Med 11:281-284. https://doi.org/10.7497/j.issn.2095-3941.2014.04.008

Li F, Qi CH, Lu YY, Hou F, Hao ZH, Zhai XC, Pang SJ (2017a) Highgrade appendiceal mucinous neoplasm associated with pseudomyxoma peritonei: a clinicalpathologic analysis of 9 cases. J Diag Pathol 24(406-410):422

Li F, Qi CH, Lu YY, Hou F, Hao ZH, Zhai XC, Pang SJ (2017b) Pseudomyxoma peritonei of non-appendiceal origin: a clinicalpathologic analysis of eight cases. J Diag Pathol 24:86-91

Li XB, Lin YL, Ji ZH, Li Y (2018) Cytoreductive surgery plus hyperthermic intraperitoneal chemotherapy in patients with pseudomyxoma peritonei: analysis of 182 patients at a single center. Chin J Clin Oncol 45:943-949

Li Y, Xu HB, Peng Z, Cui SZ, Wu W (2019) Chinese expert consensus on cytoreductive surgery and hyperthermic intraperitoneal chemotherapy for pseudomyxoma peritonei. Natl Med J China 99:1527-1535

Liu X et al (2014) Molecular profiling of appendiceal epithelial tumors using massively parallel sequencing to identify somatic mutations. Clin Chem 60:1004-1011. https://doi.org/10.1373/clinc hem.2014.225565

Mantovani G, Ballare E, Giammona E, Beck-Peccoz P, Spada A (2002) The gsalpha gene: predominant maternal origin of transcription in human thyroid gland and gonads. J Clin Endocrinol Metab 87:4736-4740. https://doi.org/10.1210/jc.2002-020183

McKenney JK, Longacre TA (2008) Low-grade mucinous epithelial neoplasm (intestinal type) arising in a mature sacrococcygeal teratoma with late recurrence as pseudomyxoma peritonei. Hum Pathol 39:629-632. https://doi.org/10.1016/j.humpa th.2007.08.021

Mittal R, Chandramohan A, Moran B (2017) Pseudomyxoma peritonei: natural history and treatment. Int J Hyperth 33:511-519. https:// doi.org/10.1080/02656736.2017.1310938

Mohamed F, Gething S, Haiba M, Brun EA, Sugarbaker PH (2004) Clinically aggressive pseudomyxoma peritonei: a variant of a histologically indolent process. J Surg Oncol 86:10-15. https://doi. org $/ 10.1002 /$ jso. 20038

Nishikawa G et al (2013) Frequent GNAS mutations in low-grade appendiceal mucinous neoplasms. Br J Cancer 108:951-958. https ://doi.org/10.1038/bjc.2013.47

Noguchi R et al (2015) Molecular profiles of high-grade and low-grade pseudomyxoma peritonei. Cancer Med 4:1809-1816. https://doi. org/10.1002/cam4.542

Nonaka D, Kusamura S, Baratti D, Casali P, Younan R, Deraco M (2006) CDX-2 expression in pseudomyxoma peritonei: a clinicopathological study of 42 cases. Histopathology 49:381-387. https ://doi.org/10.1111/j.1365-2559.2006.02512.x

Nummela P et al (2015) Genomic profile of pseudomyxoma peritonei analyzed using next-generation sequencing and immunohistochemistry. Int J Cancer 136:E282-289. https://doi.org/10.1002/ ijc. 29245

O'Connell JT, Hacker CM, Barsky SH (2002a) MUC2 is a molecular marker for pseudomyxoma peritonei. Mod Pathol 15:958-972. https://doi.org/10.1097/01.Mp.0000026617.52466.9f

O'Connell JT, Tomlinson JS, Roberts AA, McGonigle KF, Barsky SH (2002b) Pseudomyxoma peritonei is a disease of MUC2expressing goblet cells. Am J Pathol 161:551-564. https://doi. org/10.1016/s0002-9440(10)64211-3

Pengelly RJ et al (2018) Analysis of mutation and loss of heterozygosity by whole-exome sequencing yields insights into pseudomyxoma peritonei. J Mol Diagn JMD 20:635-642. https://doi. org/10.1016/j.jmoldx.2018.05.002
Pietrantonio $\mathrm{F}$ et al (2016a) GNAS mutations as prognostic biomarker in patients with relapsed peritoneal pseudomyxoma receiving metronomic capecitabine and bevacizumab: a clinical and translational study. J Transl Med 14:125. https://doi.org/10.1186/s1296 7-016-0877-x

Pietrantonio F et al (2016b) Toward the molecular dissection of peritoneal pseudomyxoma. Ann Oncol 27:2097-2103. https://doi. org/10.1093/annonc/mdw314

Pylayeva-Gupta Y, Grabocka E, Bar-Sagi D (2011) RAS oncogenes: weaving a tumorigenic web. Nat Rev Cancer 11:761-774. https ://doi.org/10.1038/nrc3106

Rose AS, Bradley AR, Valasatava Y, Duarte JM, Prlic A, Rose PW (2018) NGL viewer: web-based molecular graphics for large complexes. Bioinformatics 34:3755-3758. https://doi.org/10.1093/ bioinformatics/bty419

Saarinen L et al (2017) Multiple components of PKA and TGF-beta pathways are mutated in pseudomyxoma peritonei. PLoS ONE 12:e174898. https://doi.org/10.1371/journal.pone.0174898

Semino-Mora C, Liu H, McAvoy T, Nieroda C, Studeman K, Sardi A, Dubois A (2008) Pseudomyxoma peritonei: is disease progression related to microbial agents? A study of bacteria, MUC2 AND MUC5AC expression in disseminated peritoneal adenomucinosis and peritoneal mucinous carcinomatosis. Ann Surg Oncol 15:1414-1423. https://doi.org/10.1245/s10434-007-9778-9

Singhi AD et al (2014) GNAS is frequently mutated in both low-grade and high-grade disseminated appendiceal mucinous neoplasms but does not affect survival. Hum Pathol 45:1737-1743. https:// doi.org/10.1016/j.humpath.2014.04.018

Sio TT, Mansfield AS, Grotz TE, Graham RP, Molina JR, Que FG, Miller RC (2014) Concurrent MCL1 and JUN amplification in pseudomyxoma peritonei: a comprehensive genetic profiling and survival analysis. J Hum Genet 59:124-128. https://doi. org/10.1038/jhg.2013.132

Smeenk RM, van Velthuysen ML, Verwaal VJ, Zoetmulder FA (2008) Appendiceal neoplasms and pseudomyxoma peritonei: a population based study. Eur J Surg Oncol 34:196-201. https://doi. org/10.1016/j.ejso.2007.04.002

Tokunaga $\mathrm{R}$ et al (2019) Molecular profiling of appendiceal adenocarcinoma and comparison with right-sided and left-sided colorectal cancer. Clin Cancer Res. https://doi.org/10.1158/1078-0432. Ccr-18-3388

Velcich A, Augenlicht LH (1993) Regulated expression of an intestinal mucin gene in HT29 colonic carcinoma cells. J Biol Chem 268:13956-13961

Weinstein LS, Yu S, Warner DR, Liu J (2001) Endocrine manifestations of stimulatory $\mathrm{G}$ protein alpha-subunit mutations and the role of genomic imprinting. Endocr Rev 22:675-705. https://doi. org/10.1210/edrv.22.5.0439

Wu J et al (2011) Recurrent GNAS mutations define an unexpected pathway for pancreatic cyst development. Sci Transl Med 3:92ra66. https://doi.org/10.1126/scitranslmed.3002543

Yan FC, Li XB, Lin YL, Yu CK, Zhou Q, Li Y (2019) Clinicopathological analysis of ovarian pseudomyxoma peritonei Chin. J Clin Oncol 46:887-890

Yan FC, Lin YL, Zhou Q, Chang H, Li Y (2020) Pathological prognostic factors of pseudomyxoma peritonei: comprehensive clinicopathological analysis of 155 cases. Hum Pathol. https://doi. org/10.1016/j.humpath.2019.12.008

Publisher's Note Springer Nature remains neutral with regard to jurisdictional claims in published maps and institutional affiliations. 